\title{
Isolation of Streptococcus pneumoniae from the sputum samples and their antimicrobial resistance in Biratnagar, Nepal
}

\begin{abstract}
Sputum is one of the best specimens for the isolation of respiratory tract infections pathogen. Ten sputum specimens were collected from different hospitals of Biratnagar city where patients of age group 40-73 years suffering from headache, coughing, mild fever, chest pain had visited. In this study, a pathogen of pneumococcal disease was isolated from the sputum sample in 5\% goat blood agar by quadrant streak method. The predominant bacterial pathogen isolated from sputum sample was 10\% Streptococcus pneumoniae and $90 \%$ S. viridian. An old patient of 73 years of age was identified positive with pneumococcal disease with organisms like $S$. pneumoniae and $S$. viridian. These pathogens were identified by various biochemical test likewise, catalase, optochin, and bile solubility. S. pneumoniae isolate exhibits high sensitivity against chloramphenicol and amoxicillin but resistance against cefotaxime and erythromycin.
\end{abstract}

Keywords: sputum, Streptococcus pneumoniae, S. viridian, Biratnagar, resistance
Volume 7 Issue 6 - 2019

\author{
Sanjay Mahato, ${ }^{1,2}$ Hema Kumari Sah, ${ }^{2}$ Sudhir \\ Yadav $^{2}$ \\ 'AASRA Research and Education Academy Counsel, Nepa \\ ${ }^{2}$ Department of Microbiology, Mahendra Morang Adarsha \\ Multiple Campus, Tribhuvan University, Nepal
}

\begin{abstract}
Correspondence: Sanjay Mahato, AASRA Research and Education Academy Counsel, Biratnagar-6, Nepal, Tel +9779865658585, Email mahato.sanjay@gmail.com
\end{abstract}

Received:September 27, 2019 | Published: November 26, 2019

\section{Introduction}

Streptococcus pneumoniae (Previously Pneumococcus or Diplococcus pneumoniae) may occur intracellular or extracellular as gram-positive lanceolate diplococci but can also occur as single cocci or in short-chain of cocci. S. pneumoniae is a fastidious bacterium, which grows best at $35-37^{\circ} \mathrm{C}$ with $\square 5 \% \mathrm{CO}_{2}{ }^{1}{ }^{1}$ It is generally cultured on media that contain blood but can also be grown on a chocolate plate agar. On blood agar plate, colonies of $S$. pneumoniae appear as small, grey, moist (sometime mucoidal), and typically producing a zone of $\alpha$-hemolysis (green in color). ${ }^{1}$ The $\alpha$-hemolytic property differentiates this organism from many species, but not from viridians streptococci. It is difficult as young pneumococcal colonies appear raised similar to viridians streptococci, however, once the pneumococcal culture ages 24-48 hours, the colonies become flattened, and not occur with viridians streptococci. ${ }^{2}$ In a gram-stain,

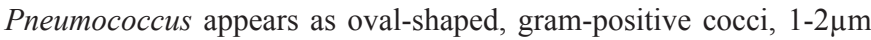
in diameter, typically in pairs, sometimes singly or short chains. ${ }^{3}$ It is catalase-negative and facultative anaerobic but can grow aerobically. Thus, it is recommended that cultures be incubated in a $\mathrm{CO}_{2}$ enriched atmosphere. ${ }^{4}$ Susceptibility to optochin (ethylhydrocuprine) and bile solubility are used to differentiate Streptococcus pneumoniae from the other viridians streptococci. Optochin is an antibacterial agent not used in therapy but only for the differentiation of Streptococcus. ${ }^{5}$

Bacterial colonization begins immediately after birth and continues throughout life with minor changes. Normal nasopharyngeal bacterial flora develops during the first year of life and the number of bacterial species varies much. ${ }^{6}$ Normal flora plays a crucial role in the prevention of infectious diseases. However, a major part of infections is caused by microbes likes $S$. pneumoniae, $H$. influenza, $N$. meningitis and Staphylococcus aureus, which originally belong to normal flora. ${ }^{7}$

Infections caused by $S$. pneumoniae can be divided into two categories mucosal infection such as otitis media and sinusitis and invasive infection such as septicemia, meningitis, and pneumonia. It belongs to the normal nasopharyngeal microbial flora that consists of bacteria with physiologic and genetic properties suitable for colonization and multiplication under certain conditions. ${ }^{8}$ The spectrum of pneumococcal disease differs in different age groups and different populations. Several risk factor for pneumococcal infection, such as age, race, immunodeficiency, other illness, socio-economic status, previous antibiotic therapy, and day-care attendance have been reported. ${ }^{9}$ The serious pneumococcal infections such as pneumonia, bacteremia, meningitis, and otitis media remain major causes of morbidity and mortality in persons of all age's worldwide. ${ }^{10}$ Capsules contribute to the ability of pneumococci to cause disease by providing resistance to phagocytosis and promoting evasion of the host immune system by the bacteria. ${ }^{11}$

Adult mortality rates for bacteremic pneumococcal pneumonia range from $10 \%$ to $30 \%$ and for meningitis from $16 \%$ to $37 \%$. Mortality rates are substantially higher in the elderly and in patients with comorbidities. ${ }^{12,13}$ Importantly, mortality rates are much higher in young children in developing countries $10 \%$ to $40 \%$, likely due to poorer access to health care and comorbidities, particularly HIV infection and malnutrition. ${ }^{14,15}$ A recent study of invasive pneumococcal disease (IPD) cases in Denmark confirmed that age and comorbidities influence survival. Mortality rates were $<3 \%$ for children younger than 5 years of age, 14\% for individuals aged 5-65 years of age, $24 \%$ for adult $\geq 65$ years of age. ${ }^{16}$

The emergence of drug-resistant Streptococcus pneumoniae will make these common infections more difficult to treat, as isolates resistant to one or more first-line agents are common in many parts of the world. ${ }^{17}$ Since the first report of decreased susceptibility to penicillin in 1967, resistance of Streptococcus pneumoniae to this drug, as well as other antibiotics, has been spread from country to country. ${ }^{18}$ Pneumococcal vaccines have been developed to prevent pneumococcal infections. Susceptibility to the macrolides was reduced among penicillin-resistant isolates. ${ }^{19}$ The lack of study and information in public domain had been a big reason for conducting this study regarding increasing pneumococcal resistance to commonly used antibiotics and its prevalence. 


\section{Methodology}

\section{Sample collection/research design}

This cross-sectional observational study was conducted in the eastern region of Nepal at three centers of Biratnagar city (Nepal) namely Koshi Zonal Hospital, Golden Hospital and Nepal Anti Tuberculosis Association (NATA) for duration of ten months (March 2017 to December 2017). The informed consent was taken from the patients. The study was approved by Mahendra Morang Adarsh Multiple Campus (MMAMC), Biratnagar and was technically supported by AASRA Research and Education Academy Counsel, Biratnagar. The study was carried out in the Microbiology Laboratory of MMAMC, Biratnagar, Nepal. A total of ten patients were tested during the study period. Demographic profile like age, gender and symptom of the patients was taken. The mouth of patient was rinsed with water. Deep breath was taken through mouth and coughed up mucous with deep coughing. To avoid contamination, each patient/ individual was instructed on how to collect a cough specimen by laboratory personnel. The specimen was collected in a $20 \mathrm{~mL}$ sterile screw-capped and wide-mouthed universal container. The container with the specimen was appropriately labeled with a unique sample number, date, and time of collection. After collection, it was forwarded to the Microbiology laboratory of MMAMC for culture and drug susceptibility testing. The specimen was analyzed within 2 hours after collection.

\section{Whole goat blood collection and defibrination}

Infection free goat was selected for blood collection. ${ }^{20}$ The goat head was in halter position so that it was slightly elevated and drawn from the side opposite to jugular vein. The vein puncture area (approximately $100 \mathrm{~cm}^{2}$ ) was disinfected with $70 \%$ alcohol. The vein was occulated by applying hand pressure in the juglar groove located in the lower neck. Goat blood is obtained by venipuncture via the jugular vein. The blood was withdrawn and collected in sterile conical flask containing sterile glass beads. Defibrination of whole goat blood was done using glass beads. ${ }^{21}$ The glass beads were soaked in $1 \mathrm{M} \mathrm{HCl}$ for overnight. Nearly $10-20 \mathrm{~g}$ of the glass beads of $4 \mathrm{~mm}$ size was placed in the $250 \mathrm{ml}$ of conical flask and was sterilized by autoclaving. $100 \mathrm{ml}$ of goat blood was measured and added aseptically to the flask of beads/tiles and sealed tightly. The conical flask was shaken gently. When the process was completed, the sound of the beads in a conical flask was changed from clear to dull. The defibrinated whole blood was aspirated aseptically and separated.

\section{Sample processing and isolation of microbes}

For the preparation of 5\% blood agar, $7.4 \mathrm{~g}$ of blood agar base powder (Himedia, Mumbai, India) was added in $95 \mathrm{ml}$ of distilled water and was sterilized by autoclaving. ${ }^{1}$ The media was cooled to $45^{\circ} \mathrm{C}$ keeping care that it didn't start solidifying. Five milliliters of the defibrinated blood was added aseptically in the blood agar base with constant shaking in such a way that forth formation prevented. The blood agar was poured in sterile Petri-plates and was solidified. After solidification of media, specimen inoculated by quadrant streak method. Then plates were incubated in candle jar at $37^{\circ} \mathrm{C}$ for $18-24$ hours. After incubation, all the plates were observed. S. pneumoniae and viridans streptococci showed $\alpha$ - hemolytic in $5 \%$ blood agar.

\section{Conventional identification of S. pneumococcus}

Gram staining and catalase test were performed on the isolates grown for $18-24$ hours on a blood agar plate at $37^{\circ} \mathrm{C}$ with approximately $5 \% \mathrm{CO}_{2}$ (or in a candle-jar). ${ }^{1}$ Gram staining was performed on the selected colonies. Gram-positive cocci bacteria with linear or irregular arrangement was considered as possible streptococci. A negative catalase test further confirmed the isolate to be streptococci. For catalase test, a pinch of bacterial colony was taken with the help of a sterile disposable loop and was mixed in $3 \% \mathrm{H}_{2} \mathrm{O}_{2}$ to the grease free slide. Immediate effervescence of bubble on the slide indicates positive test for catalase. ${ }^{22}$

Optochin test was done to confirm streptococci. ${ }^{1,22}$ The strain to be tested was grown on a blood agar plate (BAP) at $37^{\circ} \mathrm{C}$ for 18 24 hours with $\square 5 \% \mathrm{CO}_{2}$ (or in a candle-jar). A disposable loop was used to remove an isolated colony from the overnight culture on the BAP and streak on to one half of a BAP. An optochin disc $(5 \mu \mathrm{g})$ was placed within the streaked area of the BAP and incubated overnight at $37^{\circ} \mathrm{C}$ with $\square 5 \% \mathrm{CO}_{2}$. The growth on the BAP near the optochin disc was observed and the zone of inhibition was measured. A zone of inhibition of $14 \mathrm{~mm}$ or greater was indicated sensitive and was considered for presumptive identification of pneumococcus.

Bile solubility test: Ten grams of sodium deoxycholate was dissolved into $100 \mathrm{ml}$ sterile distilled water. ${ }^{1,22}$ A loopful of $10 \%$ deoxycholate solution was placed on overnight grown prospective $S$. pneumoniae colony on BAP. The colony lysed within a few minutes confirmed the isolate as $S$. pneumoniae.

\section{Antibiotic susceptibility study}

For antimicrobial testing of streptococci, 5\% defibrinated sterile goat blood was aseptically mixed to molten Mueller-Hinton Agar before plating. Antibiotic susceptibility testing of streptococci was performed by disc diffusion method (Kirby-Bauer method) on Muller-Hinton agar (Himedia, Mumbai, India) supplemented with 5\% defibrinated sterile blood and was interpreted according to the Clinical Laboratory Standard Institute (CLSI) guidelines. ${ }^{23} \mathrm{~A}$ homogenous suspension of 0.5 MacFarland standard of a pure colony (not more than 48 hours) was prepared in $5 \mathrm{~mL}$ of sterile normal saline $(0.85 \% \mathrm{NaCl})$. Using a sterile swab, the bacterial suspension was evenly distributed over the entire surface of blood supplemented MHA plates. The antibiotic disc (Himedia, Mumbai, India) containing the following antibiotics

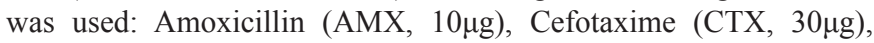

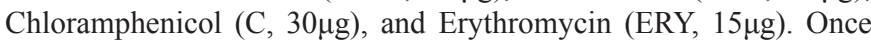
the discs were applied onto the plates, the plates were incubated at 37 ${ }^{\circ} \mathrm{C}$ for $24 \mathrm{hr}$. Zone of inhibition was measured and interpreted using the standard chart and the organisms were reported as susceptible, intermediate, or resistant accordingly.

\section{Result}

The results were obtained from sputum of more than 40 years old patients of Koshi Zonal Hospital, Golden Hospital, and NATA at Biratnagar who was admitted for a week and were suffering from fever, headache, coughing, and chest pain. When ten samples were cultured on $5 \%$ blood agar, only one sample $\left(\mathrm{H}_{1}\right)$ showed $\alpha$-hemolytic colonies of $S$. pneumoniae and $S$. viridans; while $\gamma$ - hemolytic colonies were seen in rest of the nine sample. None of the samples had $\beta$-hemolytic colonies. Out of 10 samples, $10 \%(\mathrm{n}=1)$ was $\alpha$-hemolytic and $90 \%$ $(n=9)$ were $\gamma$-hemolytic streptococci. In blood agar, $\alpha$-hemolysis showed greenish coloration around the colonies; while in $\gamma$ - hemolysis no zone was observed (Figure $1 \& 2$ ).

These isolated colonies were identified by conventional 
identification methods like Gram staining, catalase test, optochin sensitivity test, and bile solubility test. Colonies which were round, white grey with smooth margin, $1 \mathrm{~mm}$ in diameter, and $0.5 \mathrm{~mm}$ zone of $\alpha$-hemolysis showed optochin sensitivity and bile solubility (Figure 3 \& 4). This concluded the colonies were $S$. pneumoniae. Some other specific colonies which were round, white grey, but, with rough margin, having $1.5 \mathrm{~mm}$ size, and 1-2 mm zone of $\alpha$-hemolytic didn't show optochin sensitivity and bile solubility. Thus, $S$. viridans was identified.

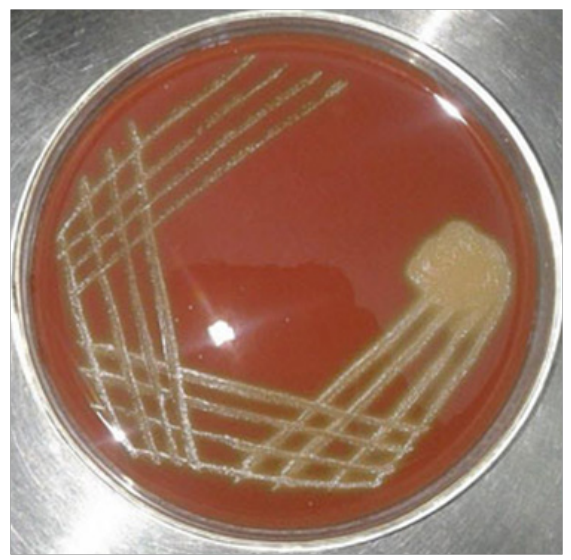

Figure I $\alpha$-hemolytic colonies of S. pneumoniae.

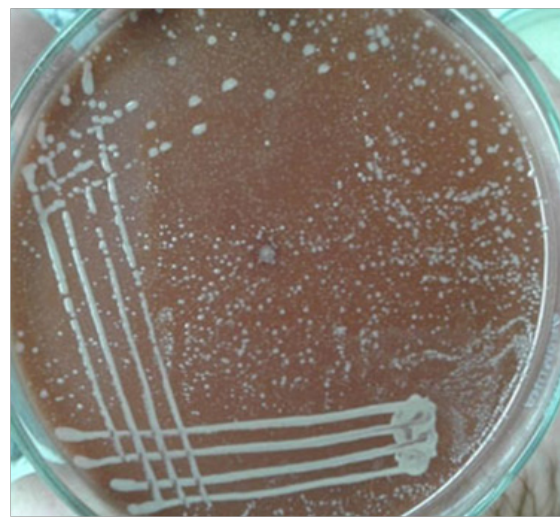

Figure $2 \gamma$-hemolytic colonies streptococci.

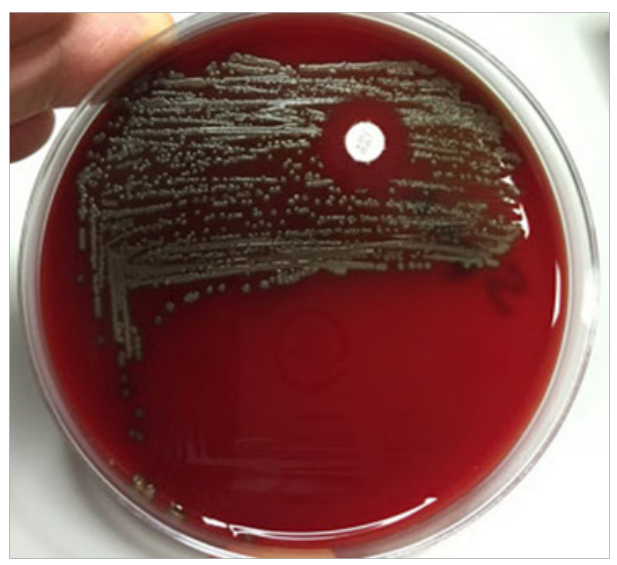

Figure 3 Optochin sensitivity on S. pneumoniae.
Antibiotic susceptibility test showed that $S$. pneumoniae was sensitive to amoxicillin and chloramphenicol, but, was resistant to cefotaxime and erythromycin (Figure $5 \& 6$ ).

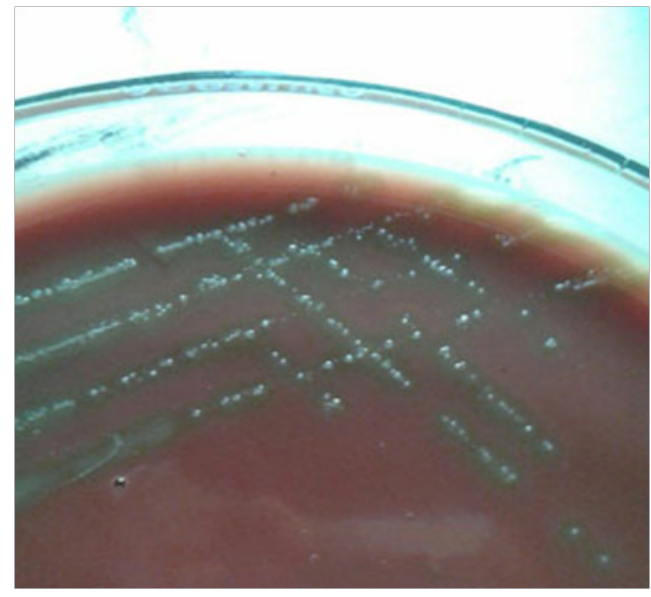

Figure 4 Bile solubility test on blood agar.

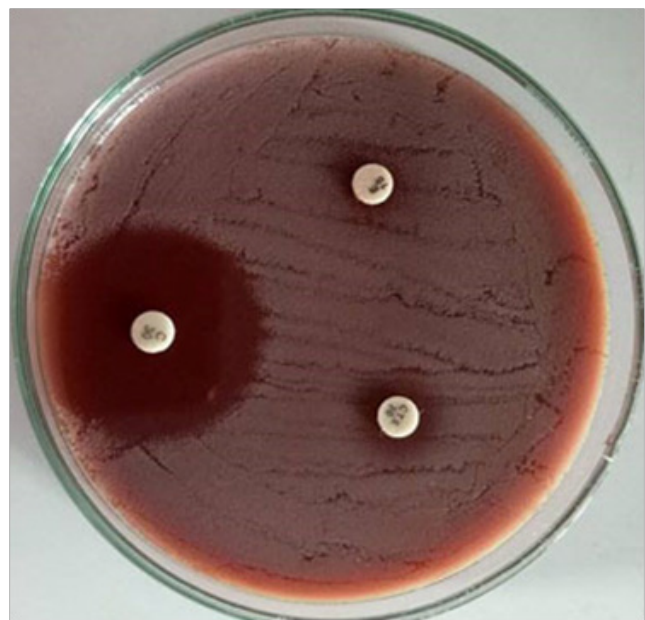

Figure $\mathbf{5}$ Antibiotic susceptibility test (AST) of S. pneumoniae.

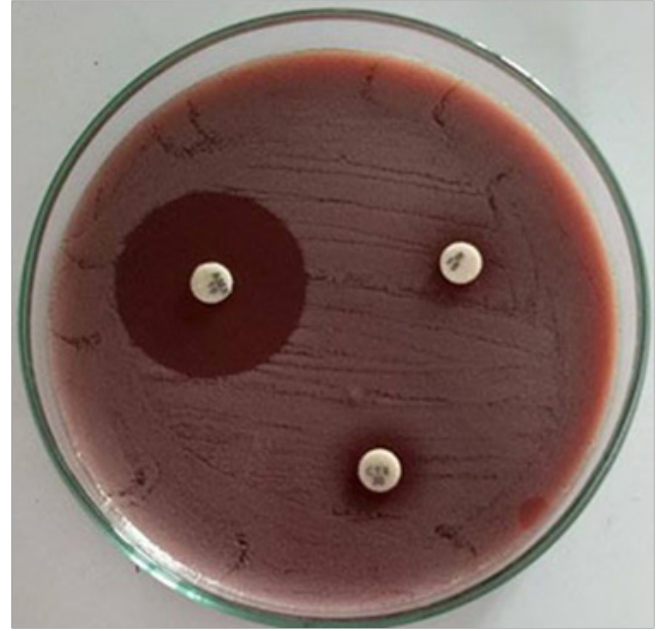

Figure 6 AST of S. pneumoniae. 
Table I Colony character and biochemical tests of S. pneumoniae and S. viridian in blood agar

\begin{tabular}{|c|c|c|}
\hline \multirow{2}{*}{$\begin{array}{l}\text { Blood agarl } \\
\text { Biochemical tests }\end{array}$} & \multicolumn{2}{|l|}{$\alpha$-hemolytic colonies } \\
\hline & S. pneumoniae & S. viridans \\
\hline Shape & Round initially, latter draughtman's colony. & $\begin{array}{l}\text { Round initially, latter no change } \\
\text { occurs in colony. }\end{array}$ \\
\hline Size & Imm & $1.5 \mathrm{~mm}$ \\
\hline Zone of $\alpha$-haemolysis & $0.5 \mathrm{~mm}$ & $\mathrm{I}-2 \mathrm{~mm}$ \\
\hline Margin & Smooth & Rough \\
\hline Opacity & Opaque & Opaque \\
\hline Colour & White-grey colony & White-grey colony \\
\hline Gram stain & +ve (lanceolate cocci) & + ve (cocci) \\
\hline Catalase & -ve & -ve \\
\hline Optochin sensitivity & Sensitive & Resistant \\
\hline Bile solubility & $+\mathrm{ve}$ & -ve \\
\hline
\end{tabular}

\section{Discussion}

When specimen was cultured on $5 \%$ blood agar, $\alpha$-hemolytic colonies were observed. Out of 10 samples, $10 \%(n=1) \alpha$-hemolytic and $90 \%(n=9) \gamma$-hemolytic streptococci were found. The findings of this study were slightly deviated from the study of Choi et al., ${ }^{24}$ reporting 5.9\% viridans streptococci and 21.3\% S. pneumoniae. $\alpha$-hemolysis showed greenish coloration around the colonies in blood agar. It occurred due to partial lysis of red blood cell present in blood agar. In $\gamma$ - hemolysis, there was no lysis of red blood cells and, hence. There was no zone of lysis. ${ }^{1}$ Streptococcus pneumoniae showed catalase negative test because they didn't produce catalase enzyme to break $\mathrm{H}_{2} \mathrm{O}_{2}$ into $\mathrm{H}_{2} \mathrm{O}$ and $\mathrm{O}_{2} \cdot{ }^{25}$

The identified isolate Streptococcus pneumoniae was grampositive lanceolate cell because of the binding of crystal violet to their cell structure. The cell wall of gram-positive organisms is more sensitive to dehydration by alcohol, a substance that acts to close up the normal pores of the wall as it contains thick layer of peptidoglycan and very low concentration of lipid. Under these conditions, the large complexes of crystal violet iodine cannot escape from the cells, and thus the primary color is retained by the gram-positive bacteria. ${ }^{26}$

The $\alpha$-hemolytic isolate was optochin sensitive and efficacy was accompanied by anaerobic condition. ${ }^{27}$ Optochin caused fragility of the bacterial cell membrane and lysed the bacterial cell due to changes in surface tension. In case of $S$. viridians, the optochin didn't change the surface tension and, hence, the cell didn't lyse. ${ }^{28}$ In bile solubility test, bile salt solution rapidly lysed pneumococcal colonies. Lysis depends on the presence of an autolytic amidase that cleaves the bond between alanine and muramic acid in the peptidoglycan of cell wall. ${ }^{29}$ Bile salts lower the surface tension between the bacterial cell membrane and the medium; consequently, accelerating the organism's natural autolytic process. Bile salt activates the autolytic enzyme which induces flattening of the colonies. ${ }^{30}$
The antimicrobial sensitivity test of $S$. pneumoniae isolated from $\mathrm{H}_{1}$ sample showed resistance towards cefotaxime and erythromycin; while susceptibility to chloramphenicol and amoxicillin. Karcic et al had reported $45 \%$ of resistance to erythromycin and $0.56 \%$ of resistance to chloramphenicol. ${ }^{31}$ The resistance to cefotaxime in the present study contradicted the findings of Chawla et al which showed $100 \%$ sensitivity to cefotaxime. ${ }^{32}$ Currently the antibiotic resistances pattern of $S$. pneumoniae isolate vary widely from one country to another within Asia. China, India, and Philippines' rate of erythromycin resistance is very high. ${ }^{33,34}$ Dias and Canica demonstrated a clear correlation between erythromycin resistance and high overall macrolide consumption, or conversely. ${ }^{35}$

The increased rate of erythromycin resistance can possibly be correlated with the wide use of this antibiotic in the communities because of its dose convenience, cost-effectiveness, and easy availability over the counter. ${ }^{36}$ Although multiple factors influence the emergence and spread of antimicrobial resistance, random antimicrobial consumption is one of the most important. ${ }^{37}$ The remarkable difference might have occurred because of the random use of antibiotics, the lack of antibiotic policy, and control in such countries. ${ }^{38}$

\section{Conclusion}

The study concludes that $\alpha$-haemolytic Streptococcus pneumoniae shows resistance to erythromycin, cefotaxime while remains sensitive to amoxicillin and chloramphenicol. Chloramphenicol and amoxicillin can be an effective drug for the treatment of respiratory tract infection patients.

\section{Acknowledgments}

None. 


\section{Conflicts of interest}

Authors declare that there is no conflict of interest.

\section{References}

1. Centers for Disease Control and Prevention. Laboratory Methods for the Diagnosis of Meningitis. Chapter 8: Identification and Characterization of Streptococcus pneumoniae. US: CDC; 2016. 14 p.

2. Buxton R. Blood Agar Plates and Hemolysis Protocols. American Society of Microbiology; 2005. 9 p.

3. Carr FJ. Microbiology: A Fundamental Introduction Second Edition. EC Microbiology. 2017;8(3):123-183.

4. Gera K, McIver KS. Laboratory growth and maintenance of Streptococcus pyogenes (the Group A Streptococcus, GAS). Curr Protoc Microbiol. 2013;30:9D.2.1-9D.2.

5. Gajbhiye P, Galate L. Optochin Resistance-Streptococcus pneumoniae in case of Pneumonia-A case report. IOSR Journal of Pharmacy and Biological Sciences. 2015;10(2):37-39.

6. Rodríguez JM, Murphy K, Stanton C, et al. The composition of the gut microbiota throughout life, with an emphasis on early life. Microb Ecol Health Dis. 2015;26:26050.

7. Mook-Kanamori BB, Geldhoff M, van der Poll T, et al. Pathogenesis and pathophysiology of pneumococcal meningitis. Clin Microbiol Rev. 2011;24(3):557-591.

8. Brooks LRK, Mias GI. Streptococcus pneumoniae's Virulence and Host Immunity: Aging, Diagnostics, and Prevention. Front Immunol. 2018;9:1366.

9. Saleem M, Naz M, Waris A, et al. Screening of pneumococcal pneumonia by amplification of pneumolysin gene in children visiting hospitals in lahore, pakistan. Iran J Pediatr. 2012;22(4):524-530.

10. Tan TQ. Pediatric invasive pneumococcal disease in the United States in the era of pneumococcal conjugate vaccines. Clin Microbiol Rev. 2012;25(3):409-419.

11. Andre GO, Converso TR, Politano WR, et al. Role of Streptococcus pneumoniae Proteins in Evasion of Complement-Mediated Immunity. Front Microbiol. 2017;8:224.

12. Soneji S, Metlay J. Mortality reductions for older adults differ by race/ethnicity and gender since the introduction of adult and pediatric pneumococcal vaccines. Public Health Rep. 2011;126(2):259-269.

13. Consolidated Guidelines on the Use of Antiretroviral Drugs for Treating and Preventing HIV Infection: Recommendations for a Public Health Approach. 2nd edition. Geneva: World Health Organization; 2016.

14. Rose AM, Hall CS, Martinez-Alier N. Aetiology and management of malnutrition in HIV-positive children. Arch Dis Child. 2014;99(6):546551.

15. Munthali T, Chabala C, Chama E, et al. Tuberculosis caseload in children with severe acute malnutrition related with high hospital-based mortality in Lusaka, Zambia. BMC Res Notes. 2017;10(1):206.

16. Hung IFN, Tantawichien T, Tsai YH, et al. Regional epidemiology of invasive pneumococcal disease in Asian adults: epidemiology, disease burden, serotype distribution, and antimicrobial resistance patterns and prevention. Int J Infect Dis. 2013;17(6):e364-e373.

17. Prestinaci F, Pezzotti P, Pantosti A. Antimicrobial resistance: a globa multifaceted phenomenon. Pathog Glob Health. 2015;109(7):309-318.

18. Mamishi S, Moradkhani S, Mahmoudi S, et al. Penicillin-Resistant trend of Streptococcus pneumoniae in Asia: A systematic review. Iran J Microbiol. 2014;6(4):198-210.

19. Kim L, McGee L, Tomczyk S, et al. Biological and Epidemiological Features of Antibiotic-Resistant Streptococcus pneumoniae in Preand Post-Conjugate Vaccine Eras: a United States Perspective. Clin Microbiol Rev. 2016;29(3):525-552.

20. Faye B, Bengoumi M. Hematology. In: Camel Clinical Biochemistry and Hematology. Springer: Cham; 2018.

21. Yeh E, Pinsky BA, Banaei N, et al. Hair sheep blood, citrated or defibrinated, fulfills all requirements of blood agar for diagnostic microbiology laboratory tests. PLoS One. 2009;4(7):e6141.

22. Cheesbrough M. District laboratory Practice in Tropical Countries. Part 2. Cambridge University Press; 2006. 143-157 p.

23. Clinical and Laboratory Standards Institute. Performance standards for antimicrobial susceptibility testing. 26th informational supplement. Document M100-S26. CLSI: Wayne, USA; 2016.

24. Choi SH, Cha SI, Choi KJ, et al. Clinical Characteristics of CommunityAcquired Viridans Streptococcal Pneumonia. Tuberc Respir Dis (Seoul). 2015;78(3):196-202.

25. Pericone CD, Park S, Imlay JA, et al. Factors contributing to hydrogen peroxide resistance in Streptococcus pneumoniae include pyruvate oxidase $(\mathrm{SpxB})$ and avoidance of the toxic effects of the fenton reaction. J Bacteriol. 2003;185(23):6815-6825.

26. Vidal JE, Ludewick HP, Kunkel RM, et al. The LuxS-dependent quorumsensing system regulates early biofilm formation by Streptococcus pneumoniae strain D39. Infect Immun. 2011;79(10):4050-4060.

27. Kariminik A, Motaghi MM. Evaluation of Antimicrobial Susceptibility Pattern of Streptococcus Mutans Isolated from Dental Plaques to Chlorhexidine, Nanosil and Common Antibiotics. International Journal of Life Sciences. 2015;9(2):18-21.

28. Shehadul Islam M, Aryasomayajula A, Selvaganapathy PR. A Review on Macroscale and Microscale Cell Lysis Methods. Micromachines (Basel). 2017;8(3):83.

29. Jordan S, Hutchings MI, Mascher T. Cell envelope stress response in Gram-positive bacteria. FEMS Microbiol Rev. 2008;32(1):107-146.

30. Urdaneta V, Casadesús J. Interactions between Bacteria and Bile Salts in the Gastrointestinal and Hepatobiliary Tracts. Front Med (Lausanne). $2017 ; 4: 163$

31. Karcic E, Aljicevic M, Bektas S, et al. Antimicrobial Susceptibility/ Resistance of Streptococcus Pneumoniae. Mater Sociomed. 2015;27(3):180-184.

32. Chawla K, Gurung B, Mukhopadhyay C, et al. Reporting Emerging Resistance of Streptococcus pneumoniae from India. J Glob Infect Dis. 2010;2(1):10-14.

33. Song JH, Jung SI, Ko KS, et al. High prevalence of antimicrobial resistance among clinical Streptococcus pneumoniae isolates in Asia (an ANSORP study). Antimicrob Agents Chemother. 2004;48(6):21012107.

34. Zhang Z, Chen M, Yu Y. Antimicrobial susceptibility among Streptococcus pneumoniae and Haemophilus influenzae collected globally between 2015 and 2017 as part of the Tigecycline Evaluation and Surveillance Trial (TEST). Infect Drug Resist. 2019;12:1209-1220.

35. Dias R, Caniça M. Trends in resistance to penicillin and erythromycin of invasive pneumococci in Portugal. Epidemiol Infect. 2008;136(7):928939. 
36. Kumar KLR, Ganaie F, Ashok V. Circulating Serotypes and Trends in Antibiotic Resistance of Invasive Streptococcus Pneumoniae from Children under Five in Bangalore. J Clin Diagn Res. 2013;7(12):27162720 .

37. Aslam B, Wang W, Arshad MI, et al. Antibiotic resistance: a rundown of a global crisis. Infect Drug Resist. 2018;11:1645-1658.
38. Manyi-Loh C, Mamphweli S, Meyer E, et al. Antibiotic Use in Agriculture and Its Consequential Resistance in Environmental Sources: Potential Public Health Implications. Molecules. 2018;23(4):795. 